\title{
Diffusion-Engineered Quasiparticle Multiplication for STJ Single Photon Detectors
}

\author{
Veronica A. Savu, Christopher M. Wilson, Luigi Frunzio, Daniel E. Prober, and Robert J. Schoelkopf
}

\begin{abstract}
We have designed a diffusion-engineered, singlephoton spectrometer in the optical-UV range using a superconducting tunnel junction. The optical photon is absorbed in a Ta film and creates excess quasiparticles. These trap into an Al tunnel junction. Internal charge multiplication is achieved with backtunneling, which occurs when the residence time of the quasiparticles near the junction is longer than the tunneling time. The collected charge is a multiple of the initially created charge. We implement backtunneling by geometrically constricting the outflow of quasiparticles, with a narrow lead. The outdiffusion time is set by the geometry of the narrow lead. Our geometry optimizes the energy resolution and count rate, while reducing the heating and noise seen with much longer confinement time. Long confinement times produce excess heating and noise, as we observed previously with quasiparticle confinement achieved via bandgap engineering.
\end{abstract}

Index Terms-Backtunneling, optical detectors, photon counting, superconducting tunnel junctions.

\section{INTRODUCTION}

$\mathbf{S}$ INGLE-PHOTON spectrometers based on superconducting tunnel junctions (STJs) have been successfully used in astronomy in the optical range, and in the EUV through soft x-ray region for synchrotron studies [1]. These applications and biological microscopy applications would benefit if the STJ based photon detectors could achieve larger count rates, and were produced in larger arrays with multiplexable low-temperature readouts. This paper describes two recent developments in that direction, arising from our studies of the x-ray and visible photon response [2]-[4]. We describe a new method to achieve a controlled amount of internal charge multiplication, using engineering of the quasiparticle outdiffusion, and we demonstrate an on-chip magnetic field control line as may be needed for larger arrays. A separate presentation (John D. Teufel, poster 4EJ06, this conference) outlines the achievement of a multiplexable low temperature readout for the STJ.

For the STJ detector, the initial number of quasiparticles $Q_{i}$ created by an absorbed photon in a superconductor is proportional to the photon energy. This is the basis of single photon spectroscopy with this detector. We employ a Ta absorber which is in good metallic contact with one electrode of an $\mathrm{Al} / \mathrm{AlOx} / \mathrm{Al}$

Manuscript received October 5, 2004. This work was supported by NASA and NASA-GSFC.

V. A. Savu is with Yale University, Department of Physics, New Haven, CT 06520 USA (e-mail: veronica.savu@yale.edu).

C. M. Wilson was with Yale University and is now with Chalmers University, Gothenburg, Sweden (e-mail: christopher.wilson@yale.edu).

L. Frunzio, D. E. Prober, and R. J. Schoelkopf are with Yale University, Department of Applied Physics, New Haven, CT 06520 USA (e-mail: luigi.frunzio@yale.edu; daniel.prober@yale.edu; robert.schoelkopf@ yale.edu)

Digital Object Identifier 10.1109/TASC.2005.849956
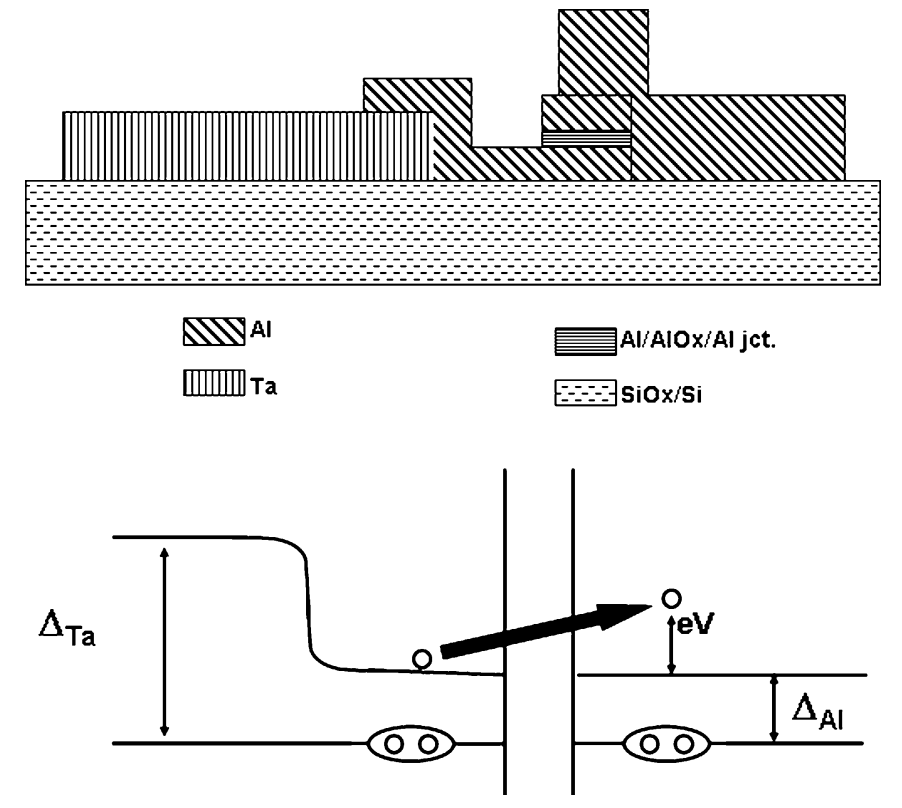

Fig. 1. Schematic geometry and energy band diagram of our nonbacktunneling detectors.

STJ. The geometry is shown schematically in Fig. 1. The superconducting tunneling current is suppressed by a parallel magnetic field. The created quasiparticles are trapped in the Al electrode, and they tunnel across the voltage-biased junction. If the quasiparticles dwell long enough in the second (counter) electrode, they can backtunnel, a process that creates current in the same direction as the direct tunneling process [5]. While the quasiparticles are confined next to the tunnel junction, they can tunnel and backtunnel a number of times, amplifying the originally created charge. The collected charge is $\mathrm{Q}=\mathrm{pQ}_{\mathrm{i}}$, with the multiplication factor $\mathrm{p}=\tau_{\text {conf }} / \tau_{\text {tunn }}$, the ratio of the confinement time to the tunnel time. This internal charge amplification process is very useful for detection in the optical and IR ranges, where each photon creates only a few thousand quasiparticles, so that $\mathrm{Q}_{\mathrm{i}}$ is very small, on the order of fC. The amplifier noise is typically a limiting factor for this small charge. The thermal quasiparticle population (i.e., that not due to visible photon absorption) also sets a limit on the detector sensitivity. We achieve a small thermal dark current by using small junctions $(1 \mu \mathrm{m} \times 2 \mu \mathrm{m}$ area), produced with electron beam lithography.

Some challenges are associated with the use of backtunneling to increase the signal-to-noise ratio. If confinement of the quasiparticles next to the junction is strong $(p \gg 1)$, the quasiparticles will tunnel and backtunnel many times; the junction heats above the bath temperature, creating excess dark current. For Al, 
$\Delta_{\mathrm{Al}}=18 \mu \mathrm{eV}$. We have found that the use of a Ta higher gap superconductor, $\Delta_{\mathrm{Ta}}=700 \mu \mathrm{eV}$, confines the quasiparticles but results in significant heating of the junction and generation of excess quasiparticles and significant noise [5], [6]. The confinement is due to bandgap engineering. Recent research at ESA [7] shows similar large heating with a Ta 'plug'. Quasiparticle confinement may also be achieved by slowing down the outdiffusion of the quasiparticles away from the junction area. We call this diffusion-engineering. The effect was clearly seen in STJ X-ray detectors with narrow Al leads that we studied previously [8]. By simply setting the width to length ratio of the $\mathrm{Al}$ outdiffusion lead, one can easily set the time the quasiparticles dwell next to the junction; then $p=\tau_{\text {diff }} / \tau_{\text {tunn }}$, with the outdiffusion time $\tau_{\text {diff }}$, and is proportional to the length to width lead ratio. We have previously presented simulations of this model and derivation of the outdiffusion time in limiting cases [9].

A second challenge appears when developing an array of STJ detectors for imaging. Even nominally identical junctions have slightly different junction dimensions. Thus, slightly different field values will be required to suppress each junction optimally. Suppressing the critical current of every STJ in an array with the same parallel field may be difficult for a big array, though the ESA experience with small arrays of relatively large junctions is encouraging. Smaller junctions are desired for lower capacitance and dark current, but these may encounter fractionally larger dimensional variations leading to larger field variations. Thus, the correct field to suppress different junctions could vary. We tried a new approach that avoids the need for a single externally-applied parallel field. We split the detector into two junctions, in a SQUID configuration, and use an Al on-chip current line that produces the needed perpendicular magnetic field. We achieve good suppression of the critical current, as required in all applications. If desired, an external magnet could still supply the correct average field for all the STJs of the array, with a small trimming field being provided to each individual STJ. If the use of an external field must be avoided entirely, the control line of each STJ can fully suppress that STJ.

\section{EXPERIMENTAL RESULTS}

Wilson et al. [5] have measured an STJ detector with a Ta plug on the counterelectrode side of the junction, and a Ta absorber that acts like a plug for quasiparticles that are trapped into the $\mathrm{Al}$ base electrode (a Nb contact, with a gap twice that of $\mathrm{Ta}$, acts to prevent outdiffusion of quasiparticles from the Ta absorber). All the measurements were done at a base temperature of $0.21 \mathrm{~K}$, in a two-stage ${ }^{3} \mathrm{He}$ Dewar. We could choose between two different photon sources. One was a mercury arc lamp, filtered to provide narrow UV lines and single photons. The other was a pulsed $\mathrm{N}_{2}$ laser. More than one photon may be absorbed in a single laser pulse, but that can be distinguished by the charge produced.

The pulse signals are read out by a current amplifier composed of a separate input 2SK146 JFET followed by an Amptek A250 transpimpedance amplifier. We add extra circuitry that allows the A250/2SK146 composite amplifier to be DC coupled and provide an active voltage bias to the junctions [10].

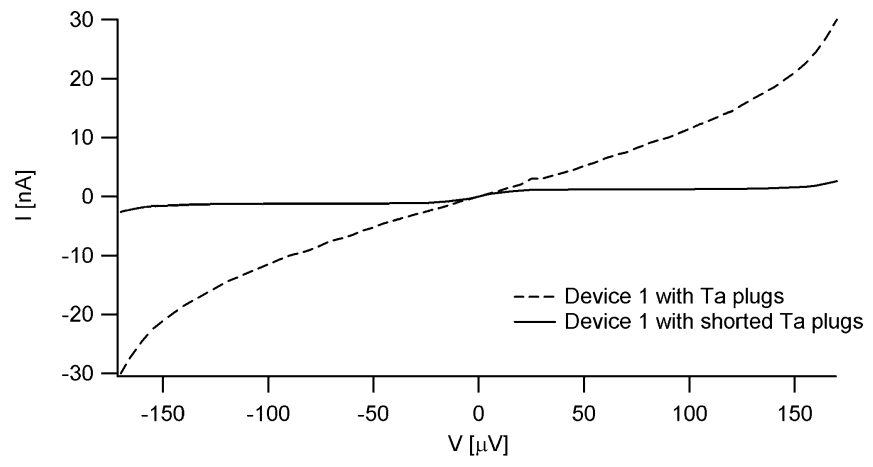

Fig. 2. I-V curves of the same device with and without Ta plugs.

The STJ devices studied had a $100 \mu \mathrm{m}^{2}$ junction and a $20 \mu \mathrm{m} \times 50 \mu \mathrm{m}$ Ta absorber; the Ta plug was the same thickness, $545 \mathrm{~nm}$, as the absorber. This device was produced with optical lithography. Fig. 2 shows the I-V curve for this device. The BCS prediction for this device is $1 \mathrm{nA}$ subgap current at $100 \mu \mathrm{V}$. We see the current is $\sim 12 \mathrm{nA}$ at $100 \mu \mathrm{V}$, and the dynamic resistance is much lower than the BCS prediction. The large excess current is due to the energy gained by the quasiparticles in the two $\mathrm{Al}$ electrodes due to the Joule $(\mathrm{I} \times \mathrm{V})$ power dissipation. The energetic quasiparticles cannot diffuse away because of the higher-gap Ta plug so each tunneling event dissipates $\mathrm{eV}$ of energy into the $\mathrm{Al}$ electrodes as seen in Fig. 1. This dissipation increases the quasiparticle population which increases the tunneling current. There is also large excess noise, which is related to the excess quasiparticle population (due to the heating) and the long recombination time of the quasiparticles [5]. Recombination times as long as $200 \mu \mathrm{s}$ were observed [11] and this leads to a charge multiplication $\mathrm{p}=\tau_{\text {recomb }} / \tau_{\text {tunn }} \sim 50-100$, with the recombination time being intrinsic and set by the quasiparticle density. This device obtains backtunneling via 'bandgap engineering' using the Ta plug.

We later did additional microfabrication on this same sample. We shorted the Ta plug with $\mathrm{Al}$ wiring, using in-situ ion beam cleaning just prior to the deposition of the Al strip that allows the quasiparticles to bypass the Ta plug. The device operation is expected to be like that of Fig. 3(a), where there is no Ta plug. Indeed, after adding the Al layer that allowed the quasiparticles to bypass the Ta plug, we obtained the expected BCS curve (Fig. 2). The resolution of this nonbacktunneling device was tested with the $\mathrm{N}_{2}$ laser. The energy resolution was $2.4 \mathrm{eV}$ full width at half maximum (fwhm). Most of this energy width is due to current noise intrinsic to this larger area junction [11]. As expected, with the Al bypass, the junction heating was absent, and the device exhibited the expected BCS I-V curve for the bath temperature. This proves that the excess current and noise are caused by the quasiparticle confinement. The long confinement time of the bandgap-engineered backtunneling device leads to large heating and noise.

Our diffusion-engineered device is designed to produce confinement in a controlled fashion that achieves $\mathrm{p} \sim 10-20$, so as to achieve significant internal charge multiplication to improve the signal-to-noise ratio with existing amplifiers, yet avoid the large heating associated with strong confinement, $\mathrm{p} \sim 100$, 

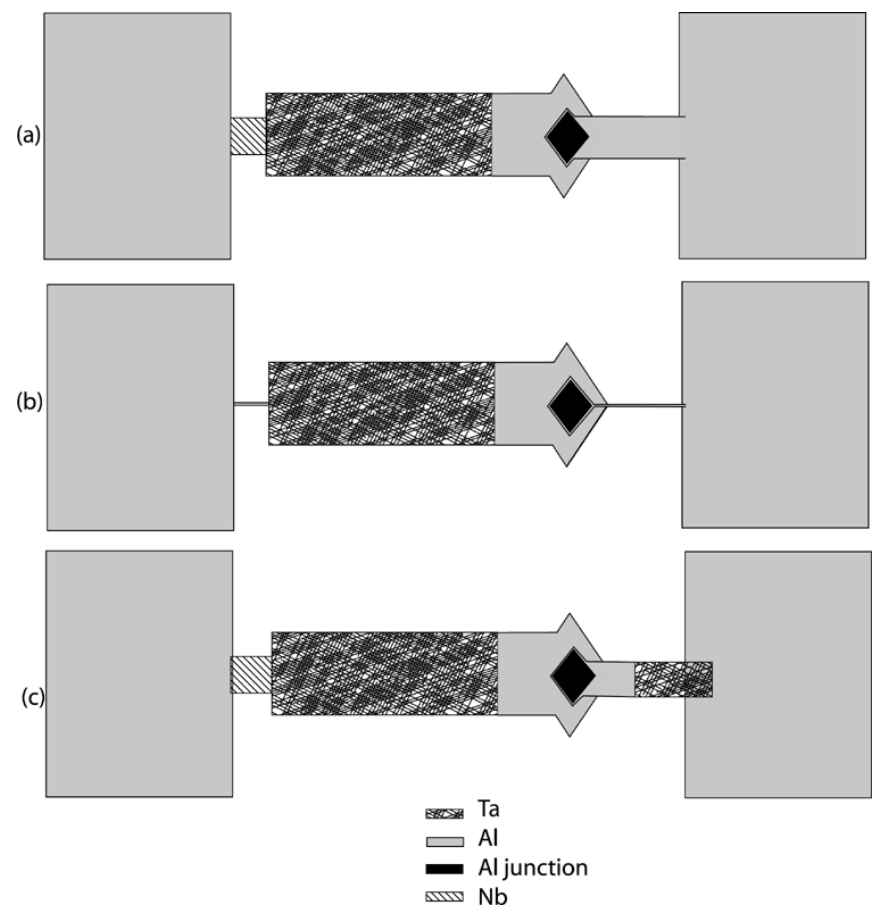

Fig. 3. Schematic geometries for the 3 cases (from top to bottom): nonbacktunneling, diffusion-engineered moderate backtunneling and band engineered strong back-tunneling devices.

achieved with the Ta plug. We can obtain a specific value of $p$ by engineering a narrow out-diffusion lead that confines the quasiparticles in the electrodes, allowing them to tunnel and backtunnel until they diffuse away [Fig. 3(a)].

In addition to using diffusion engineering to control the backtunneling, we have developed new lithographic approaches to produce micron area junctions, to reduce the dark current (proportional to junction area) and the junction capacitance. We use electron-beam lithography with angle evaporation of the junction electrodes. Such smaller junctions require a larger parallel magnetic field to suppress the critical current. Thus, we have also developed these smaller junctions in a SQUID geometry, incorporating a loop to couple in the magnetic flux to two small junctions, each of which provides tunneling. This allows the use of on-chip current leads to produce the magnetic field, which is perpendicular to the substrate. Such on-chip field control was explored in the early days of Josephson digital logic [12]. To avoid affecting the quasiparticle and phonon/equilibration properties, our control lines do not overlap the junctions.

We fabricated small Al/AlOx/Al junctions in a SQUID configuration with an on-chip control line for the perpendicular magnetic field. The area of each junction can be between 0.1 and $1 \mu \mathrm{m}^{2}$. We were able to suppress very well the critical current using the control line, to well below the sub-gap current in that experiment of $0.25 \mathrm{nA}$. An optical micrograph of two SQUIDs, fabricated from Al-AlOx-Al junctions, and the control line, is shown in Fig. 4. We have found that the required electron beam patterning of these devices is relatively straightforward, using the same ion-beam cleaning procedures developed for the junctions fabricated with optical lithography. The Al junctions are produced by angle deposition using one PMMA bilayer mask. For the optical lithography fabrication

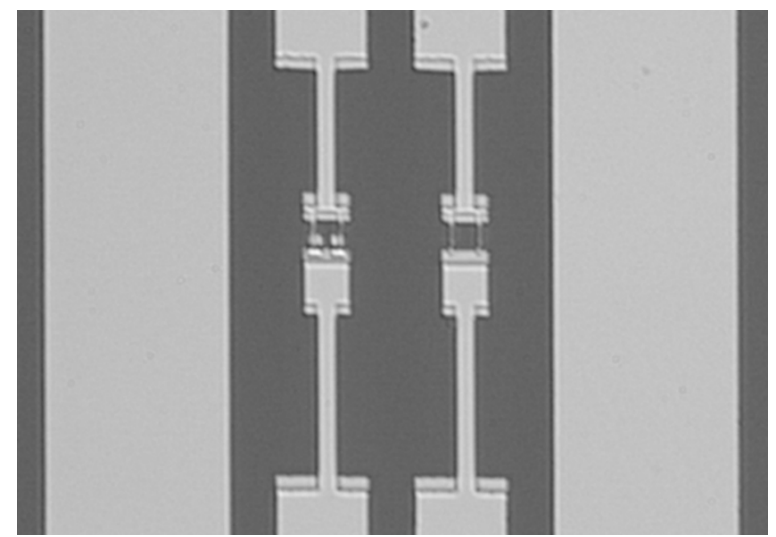

Fig. 4. Optical micrograph of 2 SQUIDs and current (magnetic field) control line.

process, the $100 \mu \mathrm{m}^{2}$ junctions were instead fabricated with an Al-AlOx-Al trilayer. It is important to note that the junction quality of the e-beam fabricated junctions is as high as obtained with the optical lithography process. However, while with the optical lithography process, junctions tested had lifetimes of at least 8 years (the length of time of testing), we do not yet have significant lifetime data for the e-beam fabrication. We are now incorporating Ta absorbers into this small junction structure, and will test the energy resolution for photon absorption. Also, although the devices shown in Fig. 4 do not incorporate the outdiffusion engineering, we have fabricated other junctions that do incorporate the diffusion engineered leads.

The small area of the $\mathrm{Al}$ trap electrodes in Fig. 4 is required by the small junction area. As a result, a very narrow lead will be required to connect the $\mathrm{Al}$ trap to the large contact, to provide the slow outdiffusion of quasiparticles. This sub-micron width lead is fabricated from $\mathrm{Al}$ with the same e-beam patterning. The Ta absorber can be large, e.g., $25 \mu \mathrm{m} \times 25 \mu \mathrm{m}$, to match the optical requirements of the intended application. The junction size and diffusion design are optimized separately from the design of the absorber.

\section{SUMMARY}

We propose a new approach to optimize the signal-to-noise ratio in the detection of optical-UV single photons using superconducting tunnel junctions. By diffusion-engineering our devices we can produce an optimal geometry that increases the signal charge yet minimizes the charge noise associated with intrinsic charge multiplication due to backtunneling.

\section{ACKNOWLEDGMENT}

The authors would like to thank J. D. Teufel, M. Shen, K. Segall, and M. Devoret for technical discussions.

\section{REFERENCES}

[1] S. Friedrich, L. J. Hiller, M. F. Cunningham, and S. E. Labov, "Linesplitting in high-resolution superconducting tunnel junction EUV detectors," IEEE Trans. Appl. Supercond., vol. 11, no. 1, pp. 836-839, Mar. 2001

[2] L. Li, L. Frunzio, and D. E. Prober, "Physical properties of the superconducting Ta film absorber of an x-ray photon detector," IEEE Trans. Appl. Supercond., vol. 13, no. 2, pp. 1124-1127, Jun. 2003. 
[3] C. M. Wilson, L. Frunzio, and D. E. Prober, "Superconducting tunnel junctions detectors for extreme ultraviolet applications," IEEE Trans. Appl. Supercond., vol. 13, no. 2, pp. 1120-1123, Jun. 2003.

[4] L. Li, L. Frunzio, C. M. Wilson, and D. E. Prober, "Quasiparticle nonequilibrium dynamics in a superconducting Ta film," J. Appl. Phys., vol. 93, no. 2, pp. 1137-1141, Jan. 2003.

[5] C. M. Wilson, L. Frunzio, and D. E. Prober, "Time-resolved measurements of thermodynamic fluctuations of the particle number in a nondegenerate Fermi gas," Phys. Rev. Lett., vol. 87, no. 067004, 2001.

[6] C. M. Wilson and D. E. Prober, "Quasiparticle number fluctuations in superconductors," Phys. Rev. B, vol. 69, no. 094524, 2004.

[7] G. Brammertz, P. Verhoeve, D. Martin, A. Peacock, and R. Venn, "Future optical detectors based on Al superconducting tunnel junctions," in SPIE Proceedings, submitted for publication.
[8] L. Li, "X-ray Single Photon Imaging Detectors Using Superconducting Tunnel Junctions," Ph.D. dissertation, Dept. of Physics, Yale Univ., New Haven, CT, 2002.

[9] V. Savu, L. Li, A. Mukherjee, C. M. Wilson, L. Frunzio, D. E. Prober, and R. J. Scheolkopf, "Diffusion-engineered single-photon spectrometer for UV/visible detection," Nuclear Instr. Methods Phys. Res. A, vol. 520, p. 237, 2004.

[10] S. Frederich et al., "Single photon imaging x-rays spectrometers using low noise current preamplifiers with dc voltage bias," IEEE Trans. Appl. Supercond, vol. 7, no. 2, pp. 3383-3386, Jun. 1997.

[11] C. M. Wilson, "Optical/UV Single-Photon Spectrometers Using Superconducting Tunnel Junctions," Ph.D. dissertation, Dept. of Physics, Yale Univ., New Haven, CT, 2002.

[12] IBM J. Res. Devel., vol. 24, no. 2, Mar. 1980. 